\title{
Geometric parametric instability of femtosecond pulses in graded-index multimode fiber
}

\author{
Uğur Teğin and Bülend Ortaç \\ National Nanotechnology Research Center (UNAM) and Institute of Materials Science and Nanotechnology, Bilkent \\ University, 06800 Bilkent, Ankara, Turkey \\ ugur.tegin@bilkent.edu.tr
}

\begin{abstract}
We numerically and experimentally study the spatio-temporal femtosecond pulse evolution in graded-index multimode fiber at normal dispersion regime. We report the first demonstration of geometric parametric instability sidebands generation with ultrashort pulses in the literature.
\end{abstract}

OCIS codes: $060.4370,190.0190$

Novel nonlinear dynamics due to spatio-temporal pulse propagation inside graded-index multimode fiber (GRIN MMF) have attracted huge attention in the recent years. Among these phenomena, geometric parametric instability (GPI) offers unrepresented frequency shift with remarkable spatial beam shape. GPI is associated with periodic refocusing inside GRIN MMFs and theoretically predicted by Longhi [1]. Later, the proposed theory is verified by experimental studies and GPI sidebands are reported with more than $100 \mathrm{THz}$ detuning from to pump frequency $[2,3]$. These studies focused on quasi- continues pulse evolution in GRIN MMF because of the analogy between GPI and the modulation instability thus the spatio-temporal evolution of femtosecond pulses at normal dispersion regime remains unknown. Here, we present the first experimental observation of GPI sidebands with femtosecond pulses in GRIN MMF. To gain detailed understanding, we perform numerical simulations and theoretical calculations and obtained results are well-aligned with experimental observations.

We perform numerical simulations by coupled-mode analysis method [4]. We launched We launch pulses with 200 fs pulse duration, $350 \mathrm{~nJ}$ pulse energy at $800 \mathrm{~nm}$. Even though GRIN MMF supports hundreds of modes at $800 \mathrm{~nm}$, to reduce computation time, we perform a simulation with linearly polarized first six cylindrically symmetric modes for $30 \mathrm{~cm}$ GRIN MMF with $50 \mu \mathrm{m}$ core diameter. The initials pulse energy is distributed among these six modes (50\% in $\mathrm{p}=0,18 \%$ in $\mathrm{p}=1,13 \%$ in $\mathrm{p}=2,10 \%$ in $\mathrm{p}=3,6 \%$ in $\mathrm{p}=4$ and $3 \%$ in $\mathrm{p}=5$ ) and obtained results are presenting first GPI sidebands with $90 \mathrm{THz}$ frequency offset from the central pump frequency (see Fig.1(a)). The pulse propagation equation used in the numerical calculations for the each fiber mode $p$ is presented as:

$$
\begin{aligned}
\frac{\partial A_{p}}{\partial z} & =i \delta \beta_{0}^{(p)} A_{p}-\delta \beta_{1}^{(p)} \frac{\partial A_{p}}{\partial t}-i \frac{\beta_{2}^{(p)}}{2} \frac{\partial^{2} A_{p}}{\partial^{2} t} \\
& +i \frac{\gamma}{3}\left(1+\frac{i}{\omega_{0}} \frac{\partial}{\partial t}\right) \sum_{l, m, n} \eta_{p l m n}\left[\left(1-f_{R}\right) A_{l} A_{m} A_{n}^{*}+f_{R} A_{l} \int h_{R} A_{m}(z, t-\tau) A_{n}^{*}(z, t-\tau) d \tau\right]
\end{aligned}
$$

where $\eta_{p l m n}$ is nonlinear coupling coefficient, $f_{R} \approx 0.18$ is the fractional contribution of the Raman effect, $h_{R}$ is the delayed Raman response function and $\delta \beta_{0}^{(p)}\left(\delta \beta_{1}^{(p)}\right)$ is difference between first (second) Taylor expansion coefficient of propagation constant for corresponding and the fundamental mode.

In experiments, we use an amplified Ti:Sapphire laser as a pump source to generate linearly polarized, single- mode, 200 femtosecond ultrashort pulses at $800 \mathrm{~nm}$ with $1 \mathrm{kHz}$ repetition rate. A GRIN MMF with $50 \mathrm{~m}$ core diameter is excited with a lens and three-axis translation stage configuration (Fig.1(b)). When we excite sufficient amount of high order modes with $354 \mathrm{~nJ}$ pump pulse, we obtain first GPI peak pair with $91 \mathrm{THz}$ frequency shift (Fig.1.(c)). First Stokes and anti-Stokes peaks are observed around $1055 \mathrm{~nm}$ and $645 \mathrm{~nm}$ with $12 \mathrm{~nm}$ and $5 \mathrm{~nm}$ spectral bandwidths, respectively. Commonly used theoretical calculation $[1,2,3]$ suggests that $89 \mathrm{THz}$ frequency detuning is expected for first GPI peak pair when pump pulses are selected at $800 \mathrm{~nm}$ which corresponds to $1049 \mathrm{~nm}$ and $646 \mathrm{~nm}$ for first GPI Stokes and anti-Stokes, respectively. Experimentally obtained spatial intensity distribution of first GPI Stokes features Gaussian shape as the pump pulse. 
(b)
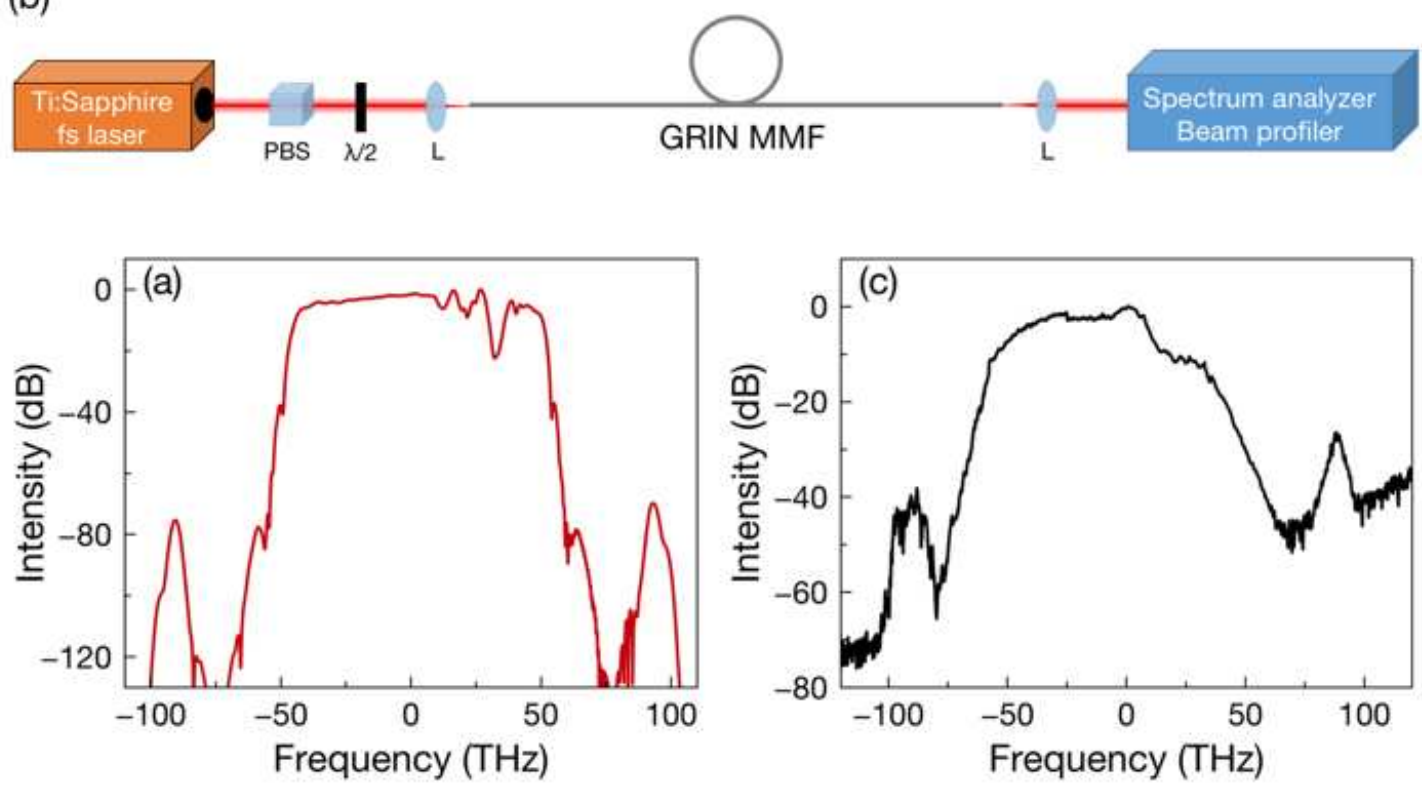

Fig. 1. (a) Numerically obtained spectrum with 345 nJ, 200 fs pulses after $30 \mathrm{~cm}$ GRIN MMF. (b) Schematic of the experimental setup. (c) Experimentally obtained spectrum with $345 \mathrm{~nJ}, 200 \mathrm{fs}$ pulses after $260 \mathrm{~cm}$ GRIN MMF.

In conclusion, we study the spatio-temporal evolution of femtosecond pulses in GRIN MMF experimentally and numerically. Our results present the first demonstration of GPI sidebands generation with ultrashort pulses in the literature. Numerical simulations and theoretical calculations validate experimental observations. Observed results provide inside of the GPI formation with ultrashort pulses to complete spatio-temporal pulse evolution studies and indicate that the known attractor effect observed in GRIN MMF for quasi-continuous pump pulses also exists for ultrashort pump pulses [5]. With unique frequency shift, GPI can be used as new wavelength generation method. Obtained frequency shifts are depending on the wavelength of the pump pulses thus it can be tuned for desired applications.

\section{References}

1. S. Longhi, "Modulational instability and space time dynamics in non- linear parabolic-index optical fibers," Opt. Lett. 28, 2363-2365 (2003).

2. K. Krupa, A. Tonello, A. Barthelemy, V. Couderc, B. M. Shalaby, A. Bendahmane, G. Millot, and S. Wabnitz, Observation of geometric parametric instability induced by the periodic spatial self-imaging of multimode waves, Phys. Rev. Lett., 116, 183901-183906 (2016).

3. S. Longhi, "Modulational instability and space time dynamics in non- linear parabolic-index optical fibers," Opt. Lett. 28, 2363-2365 (2003).

4. G. Lopez-Galmiche, Z. S. Eznaveh, M. Eftekhar, J. A. Lopez, L. Wright, F. Wise, D. Christodoulides, and R. A. Correa, Visible supercontinuum generation in a graded index multimode fiber pumped at $1064 \mathrm{~nm}$, Opt. Lett. 41, 2553-2556 (2016).

5. L. G. Wright, Z. Liu, D. A. Nolan, M.-J. Li, D. N. Christodoulides, and F. W. Wise, Self-organized instability in graded-index multimode fibres, Nat. Photon. 10, 771-775 (2016). 